\title{
COMPUTATION OF TOPOLOGICAL INDICES OF LINE GRAPH OF JAHANGIR GRAPH
}

\author{
Mehdi Alaeiyan ${ }^{\mathrm{a}}$, Muhammad S. Sardar ${ }^{\mathrm{b}}$, Sohail Zafar ${ }^{\mathrm{b}}$, \\ Zohaib Zahid ${ }^{b}$ and Mohammad R. Farahani ${ }^{a}$ \\ aDepartment of Mathematics, Iran University of Science and Technology (IUST), \\ Narmak, Tehran 16844, Iran \\ ${ }^{b}$ Department of Mathematics, University of Management and Technology (UMT), \\ Lahore, Pakistan
}

\begin{abstract}
In this paper, we compute first Zagreb index (coindex), second Zagreb index (coindex), third Zagreb index, first hyper-Zagreb index, atom-bond connectivity index, fourth atom-bond connectivity index, sum connectivity index, Randic connectivity index, augmented Zagreb index, Sanskruti index, geometric-arithmetic connectivity index and fifth geometricarithmetic connectivity index of line graph of Jahangir graph.
\end{abstract}

Keywords: topological indices, line graph, coindices.

\footnotetext{
${ }^{*}$ Corresponding author.

E-mail address: Mr_Farahani@mathdep.iust.ac.ir (Mohammad R. Farahani).

Copyright ( 2018 Scientific Advances Publishers

2010 Mathematics Subject Classification: 05C05, 05C12, 05C15, 05C31, 05 C69.

Submitted by Jianqiang Gao.

Received May 8, 2018; Revised May 15, 2018
} 


\section{Introduction and Preliminaries}

Let $G=(V ; E)$ be a simple graph, with vertex set $V$ and edge set $E \in V \times V$, on $p=|V|$ vertices and $q=|E|$ edges. The complement of a graph $G$, denoted by $G$, is a simple graph on the same set of vertices $V(G)$ in which two vertices $u$ and $v$ are connected by an edge $u v$, if and only if they are not adjacent in $G$. Obviously, $E(G) \cup E(\bar{G})=E\left(K_{p}\right)$, where $K_{p}$ is complete graph of order $p$, and $|E(\bar{G})|=\frac{p(p-1)}{2}-q$. The degree $d_{\nu}$ of a vertex $v$ is the number of vertices joining to $v$ and the degree $d_{e}$ of an edge $e \in E(G)$, where $d_{e}$ is the number of its adjacent vertices in $V(L(G))$, where $L(G)$ is the simple graph whose vertices are the edges of $G$, with $u \nu \in E(L(G)$ when $u$ and $v$ have a common end point in $G$. In structural chemistry, line graph of a graph $G$ is very useful. The first topological indices on the basis of line graph was introduced by Bertz in 1981 (see [1]). For more details on line graph, see the articles [2-7].

Topological indices are the numerical quantities which represent the structure of any simple finite graph. They are invariant under the graph isomorphisms. The idea of topological index appears from work done by Wiener (see [8]) in 1947 although he was working on boiling point of para $n$. He called this index as Wiener index and then theory of topological index started. The Wiener index of graph $G$ is defined as follows:

$$
W(G)=\frac{1}{2} \sum_{(u, v)} d(u, v)
$$

where $(u ; v)$ is any ordered pair of vertices in $G$ and $d(u ; v)$ is $u-v$ geodesic.

The Zagreb indices were first introduced by Gutman in [9], they are important molecular descriptors and have been closely correlated with many chemical properties (see [10]) and defined as: 


$$
\begin{aligned}
& M_{1}(G)=\sum_{u \in V(G)} d_{u}^{2}, \\
& M_{2}(G)=\sum_{u \nu \in E(G)} d_{u} d_{\nu} .
\end{aligned}
$$

In fact, one can rewrite the first Zagreb index as

$$
M_{1}(G)=\sum_{u \nu \in E(G)}\left[d_{u}+d_{\nu}\right]
$$

The third Zagreb index, introduced by Fath-Tabar in [11]. This index is defined as follows:

$$
M_{3}(G)=\sum_{u \nu \in E(G)}\left|d_{u}-d_{\nu}\right|
$$

The hyper-Zagreb index was first introduced in [12]. This index is defined as follows:

$$
H M(G)=\sum_{u \nu \in E(G)}\left(d_{u}+d_{\nu}\right)^{2}
$$

The first Zagrab coindex is defined as:

$$
\overline{M_{1}}=\overline{M_{1}}(G)=\sum_{u \nu \notin E(G)}\left[d_{u}+d_{\nu}\right]
$$

The second Zagrab coindex is defined as:

$$
\overline{M_{2}}=\overline{M_{2}}(G)=\sum_{u \nu \notin E(G)} d_{u} d_{\nu} .
$$

The degree distance index for graphs developed by Dobrynin and Kochetova in [13] and Gutman in [14] as a weighted version of the Wiener index. The degree distance of $G$, denoted by $D D(G)$, is defined as follows:

$$
D D(G)=\sum_{\{u, \nu\} \subseteq V(G)} d(u, \nu)\left[d_{u}+d_{\nu}\right]
$$


Randic index introduced by Randic in 1975 (see [15]). This index is defined as follows:

$$
R(G)=\sum_{u \nu \in E(G)} \frac{1}{\sqrt{d_{u} d_{\nu}}}
$$

Later, this index was generalized by Bollobas and Erdos (see [16]) to the following form for any real number, and named the general Randic index:

$$
R_{\alpha}(G)=\sum_{u \nu \in E(G)} d_{u} d_{\nu}^{\alpha}
$$

The atom-bond connectivity index (ABC), introduced by Estrada et al. in [17] which has been applied up until now to study the stability of alkanes and the strain energy of cycloalkanes. The $\mathrm{ABC}$ index of $G$ is defined as:

$$
A B C(G)=\sum_{u \nu \in E(G)} \sqrt{\frac{d_{u}+d_{\nu}-2}{d_{u} d_{\nu}}} .
$$

For more details, see the article [18]. In 2010, the general sum-connectivity index $(G)$ has been introduced in [19]. For more detail on sum connectivity, we refer the articles [20,21]. This index is defined as follows:

$$
\chi(G)=\sum_{u \nu \in E(G)} \frac{1}{\sqrt{d_{u}+d_{\nu}}} .
$$

Vukicevic and Furtula introduced the geometric arithmetic (GA) index in [22]. The GA index for $G$ is defined by

$$
G A(G)=\sum_{u \nu \in E(G)} \frac{2 \sqrt{d_{u} d_{\nu}}}{d_{u}+d_{\nu}} .
$$

Inspired by the work on the $\mathrm{ABC}$ index, Furtula et al. proposed the following modified version of the $\mathrm{ABC}$ index and called it as augmented Zagreb index (AZI) in [28]. This index is defined as follows:

$$
A Z I(G)=\sum_{u \nu \in E(G)}\left(\frac{d_{u} d_{\nu}}{d_{u}+d_{\nu}-2}\right)^{3} .
$$


The fourth member of the class of ABC index was introduced by Ghorbani et al. in [24-26] as:

$$
A B C_{4}(G)=\sum_{u \nu \in E(G)} \sqrt{\frac{S_{u}+S_{\nu}-2}{S_{u} S_{\nu}}}
$$

where $S_{u}$ is the summation of degrees of all neighbours of vertex $u$ in $G$. In other words, $S_{u}=\sum_{u \nu \in E(G)} d_{\nu}$. Similarly for $S_{\nu}$.

The 5-th GA index was introduced by Graovac et al. in [27] as:

$$
G A_{5}(G)=\sum_{u \nu \in E(G)} \frac{2 \sqrt{S_{u} S_{\nu}}}{S_{u}+S_{\nu}}
$$

The prediction power is better than the $\mathrm{ABC}$ index in the study of heat of formation for heptanes and octanes (see [27]).

The Sanskruti index $S(G)$ of a graph $G$ is defined in [29] as follows:

$$
S(G)=\sum_{u \nu \in E(G)}\left(\frac{S_{u} S_{\nu}}{S_{u}+S_{\nu}-2}\right)^{3} .
$$

Theorem 1.1 ([14]). Let $G$ be a graph of order $p$ and size $q$. Then

$$
\begin{aligned}
& M_{1}(\bar{G})=M_{1}(G)+p(p-1)^{2}-4 q(p-1) ; \\
& \overline{M_{1}}(G)=2 q(p-1)-M_{1}(G) ; \\
& \overline{M_{1}}(\bar{G})=2 q(p-1)-M_{1}(G) .
\end{aligned}
$$

Theorem 1.2 ([30]). Let $G$ be a graph of order $p$ and size $q$. Then:

$$
\begin{aligned}
& M_{2}(\bar{G})=\frac{1}{2} p(p-1)^{3}-3 q(p-1)^{2}+2 q^{2}+\frac{2 p-3}{2} \\
& M_{1}(G)-M_{2}(G) ; \overline{M_{2}}(G)=2 q^{2}-\frac{1}{2} M_{1}(G)-M_{2}(G) \\
& \overline{M_{2}}(\bar{G})=q(p-1)^{2}-(p-1) M_{1}(G)+M_{2}(G)
\end{aligned}
$$


Theorem 1.3 ([23]). Let $G$ be a graph of order $p$ and size $q$. Then

$$
\overline{M_{1}}(G) \geq 2 W(G)-2 M_{1}(G)+6 q(p-1)-p^{3}+p^{2} .
$$

Theorem 1.4 ([23]). Let $G$ be a nontrivial graph of diameter $d \geq 2$. Then

$$
\overline{M_{1}}(G) \leq \frac{D D(G)-M_{1}(G)}{2},
$$

with equality if and only if $d=2$.

The following lemma is helpful for computing the degree of a vertex of line graph.

Lemma 1.5. Let $G$ be a graph with $u ; v \in V(G)$ and $e=u \nu \in E(G)$.

Then $d_{e}=d_{u}+d_{v}-2$.

Lemma 1.6 ([31]). Let $G$ be a graph of order $p$ and size $q$, then the line graph $L(G)$ of $G$ is a graph of order $p$ and size $1 / 2 M_{1}(G)-q$.

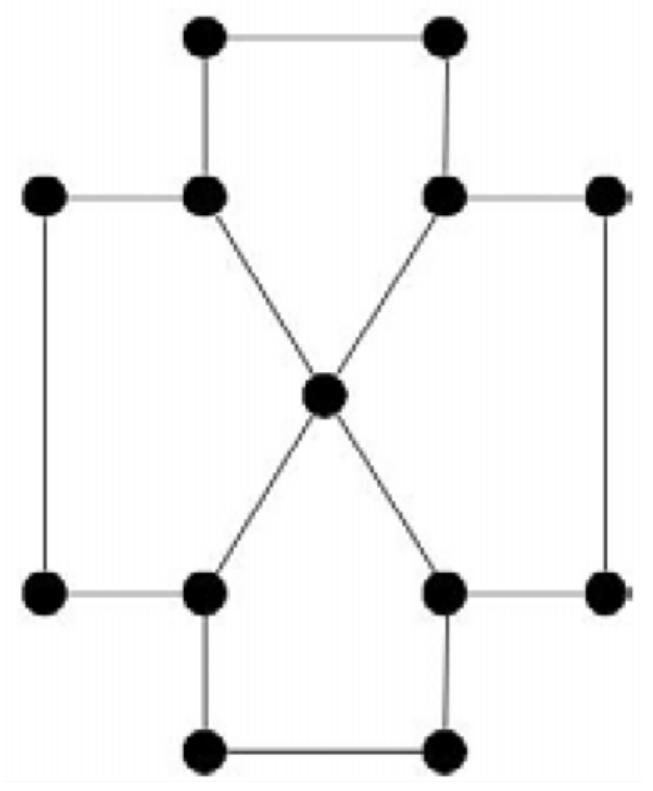

Figure 1. The Jahangir graph $J_{3 ; 4}$. 


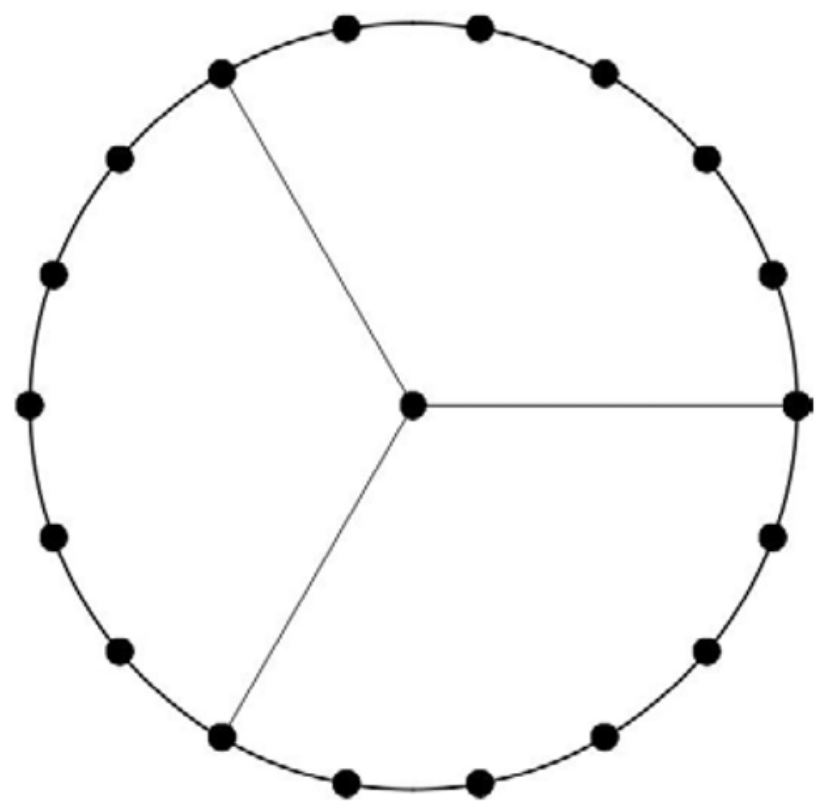

Figure 2. The Jahangir graph $J_{6 ; 3}$.

\section{Main Results and Discussions}

Jahangir graph $J_{n ; m}$ for $m \geq 3$, a graph consisting of a cycle $C_{n m}$ with one additional vertex which is adjacent to $m$ vertices of $C_{n m}$ at distance $n$ to each other on $C_{n m}$ (see [32]). The $J_{n m}$ has order $n m+1$ and size $m(n+1)$. The graphs $J_{3 ; 4}$ and $J_{6 ; 3}$ are shown in the Figures 1 and 2 , respectively.

Theorem 2.1. Let $G$ be the line graph of the Jahangir graph $J_{n ; m}$. Then

(I) $M_{3}(G)=-2 m^{2}+2 m$;

(II) $H M(G)=16 m n+38 m+2 m(4+m)^{2}+2 m(m-1)(m+1)^{2}$;

(III) $\overline{M_{1}}(G)=\overline{M_{1}}(\bar{G})=m^{3} n+2 m^{2} n^{2}+5 m^{2} n-6 m n+2 m^{3}+4 m^{2}+32 m$; 
(IV) $M_{1}(\bar{G})=m^{3} n^{3}+m^{3} n-3 m^{2} n^{2}-14 m^{2} n-4 m^{2}+9 m n+18 m$;

(V) $M_{2}(G)=4 m n+6 m^{2}+15 m+\frac{1}{2} m(m-1)(m+1)^{2}$;

(VI) $M_{2}(\bar{G})=\frac{1}{2} m(n+1)(m(n+1)-1)^{3}-\frac{3}{2}\left(m^{2}+3 m+2 m n\right)(m n+m-1)^{2}$

$$
\begin{aligned}
& +\frac{1}{2}\left(m^{2}+3 m+2 m n\right)^{2}+2(2 m n+2 m-3) m n-4 m^{2} \\
& -4 m-4 m n+m^{3}-\frac{1}{2} m(m-1)(m+1)^{2}
\end{aligned}
$$

(VII) $\overline{M_{2}}(G)=2 m^{3} n+6 m^{2} n+2 m n^{2}-6 m n+2 m^{3}-2 m^{2}-20 m$;

(VIII) $\overline{M_{2}}(\bar{G})=\frac{1}{2} m^{4} n^{2}+m^{3} n^{3}+\frac{7}{2} m^{3} n^{2}+m^{3} n-6 m^{2} n^{2}-20 m^{2} n$

$$
+9 m n-6 m^{2}+27 m
$$

(IX) $W(G) \leq\left(\frac{1}{2} m^{2}+\frac{3}{2} m+m n\right)(m(n+1)-1)+2 m n+18 m+3 m^{2}$

$$
\begin{aligned}
& +\frac{3}{2} m\left(m^{2}-1\right)-\frac{3}{2}\left(m^{2}+3 m+2 m n\right)(m n+m-1) \\
& +\frac{1}{2} m^{3}(n+1)^{3}-\frac{1}{2} m^{2}(n+1)^{2}
\end{aligned}
$$

(X) $D D(G) \geq 4\left(\frac{1}{2} m^{2}+\frac{3}{2} m+m n\right)(m(n+1)-1)-4 m n+36 m+6 m^{2}$

$$
+3 m\left(m^{2}-1\right)
$$

(XI) $A B C(G)=\frac{1}{2}(n-3) m \sqrt{2}+m \sqrt{2}+\frac{2}{3} m+\frac{2}{3} m \sqrt{3} \sqrt{\frac{2+m}{m+1}}$

$$
+\frac{1}{2} m(m-1) \sqrt{2} \sqrt{\frac{m}{(m+1)^{2}}}
$$




$$
\begin{aligned}
& \text { (XII) } R(G)=\frac{1}{2}(n-3) m+\frac{1}{3} m \sqrt{6}+\frac{1}{3} m+\frac{2 m}{\sqrt{3 m+3}}+\frac{1}{2} \frac{m(m-1)}{\sqrt{(m+1)^{2}}} ; \\
& \text { (XIII) } G A(G)=(n-3) m+\frac{4}{5} m \sqrt{6}+m+\frac{4 m \sqrt{3 m+3}}{4+m}+\frac{m(m-1) \sqrt{(m+1)^{2}}}{2 m+2} \\
& \text { (XIV) } \chi(G)=\frac{1}{2}(n-3) m+\frac{2}{5} m \sqrt{5}+\frac{1}{6} m \sqrt{6}+\frac{2 m}{\sqrt{4+m}}+\frac{1}{2} \frac{m(m-1)}{\sqrt{2 m+2}} \\
& \text { (XV) } A Z I(G)=8(n-3) m+\frac{1753}{64} m+\frac{54 m(m+1)^{3}}{(2+m)^{3}}+\frac{1}{16} \frac{m(m-1)(m+1)^{6}}{m^{3}}
\end{aligned}
$$

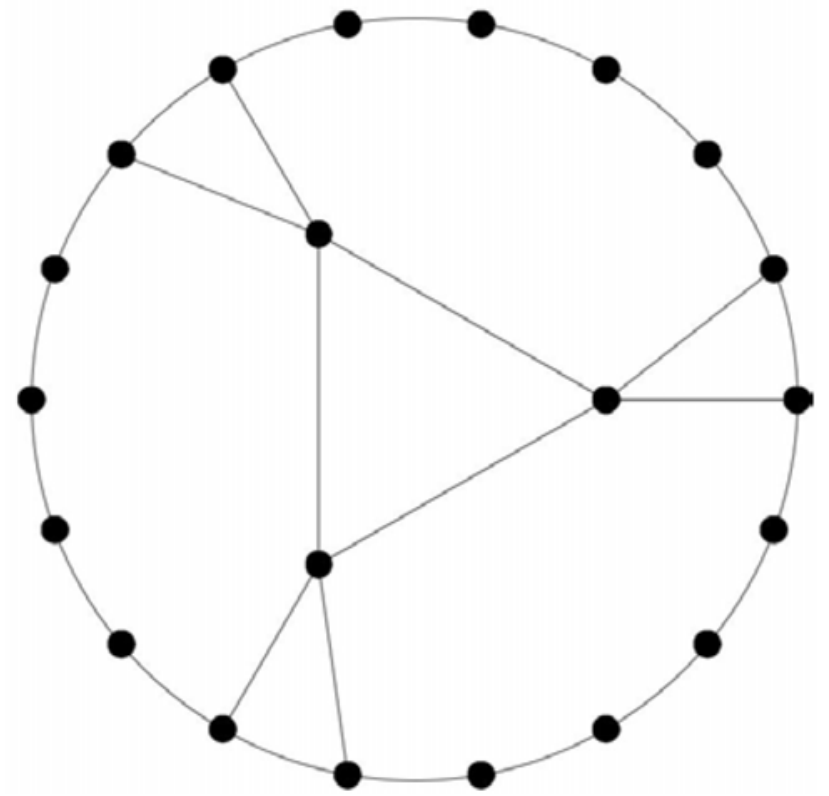

Figure 3. The line graph of Jahangir graph $J_{6 ; 3}$.

Proof. The graph $G$ for $n=6$ and $m=3$ is shown in Figure 3. By using Lemma 1.5 , it is easy to see that the order of $G$ is $m(n+1)$ out of which $2 m$ vertices are of degree $3, m$ vertices are of degree $m+1$ and $(n-2) m$ vertices are of degree 2. Therefore by using Lemma 1.6, $G$ has size 
$\frac{m^{2}+2 m n+3 m}{2}$. We partition the size of $G$ into edges of the type $E_{(d u ; d \nu)}$, where $u \nu$ is an edge. In $G$, we get edges of the type $E_{(2 ; 2)}$, $E_{(2 ; 3)}, E_{(3 ; 3)}, E_{(3 ; m+1)}$, and $E_{(m+1 ; m+1)}$. The number of edges of these types are given in the Table 1.

By using Formulas (1)-(12), Table 1 and by employing the Equations (16)-(23), we can obtain the required results.

Table 1. The size partition of $G$

\begin{tabular}{|c|c|c|c|c|c|}
\hline $\begin{array}{c}\left(d_{u}, d_{\nu}\right), \text { where } \\
u \nu \in E(G)\end{array}$ & $(2,2)$ & $(2,3)$ & $(3,3)$ & $(3, m+1)$ & $(m+1, m+1)$ \\
\hline $\begin{array}{c}\text { Number of edges } \\
\text { N }\end{array}$ & $m(n-3)$ & $2 m$ & $m$ & $2 m$ & $\frac{m(m-1)}{2}$ \\
\hline
\end{tabular}


Theorem 2.2. Let $G$ be the line graph of the Jahangir graph $J_{n ; m}$. Then

$$
\begin{aligned}
& A B C_{4}(G)= \\
& \int m \sqrt{\frac{2 m+10}{(m+6)^{2}}}+\frac{1}{2} m(m-1) \sqrt{\frac{2 m^{2}+8}{\left(m^{2}+5\right)^{2}}} \\
& +2 m \sqrt{\frac{m^{2}+m+9}{\left(m^{2}+5\right)(m+6)}}+\frac{1}{3} m \sqrt{6} \sqrt{\frac{m+10}{m+6}}, \quad \text { if } n=3 ; \\
& m \sqrt{\frac{2 m+10}{(m+6)^{2}}}+\frac{1}{2} m(m-1) \sqrt{\frac{2 m^{2}+8}{\left(m^{2}+5\right)^{2}}} \\
& +2 m \sqrt{\frac{m^{2}+m+9}{\left(m^{2}+5\right)(m+6)}}+\frac{2}{5} m \sqrt{5} \sqrt{\frac{m+9}{m+6}}, \quad \text { if } n=4 \\
& \frac{1}{5} m \sqrt{35}+m \sqrt{\frac{2 m+10}{(m+6)^{2}}}+\frac{1}{2} m(m-1) \sqrt{\frac{2 m^{2}+8}{\left(m^{2}+5\right)^{2}}} \\
& \begin{array}{l}
+2 m \sqrt{\frac{m^{2}+m+9}{\left(m^{2}+5\right)(m+6)}}+\frac{2}{5} m \sqrt{5} \sqrt{\frac{m+9}{m+6}}, \\
\text { 5) } m \sqrt{6}+\frac{1}{5} m \sqrt{35}+m \sqrt{\frac{2 m+10}{(m+6)^{2}}}+\frac{1}{2} m(m-1) \sqrt{\frac{2 m^{2}+8}{\left(m^{2}+5\right)^{2}}}
\end{array} \\
& +2 m \sqrt{\frac{m^{2}+m+9}{\left(m^{2}+5\right)(m+6)}}+\frac{2}{5} m \sqrt{5} \sqrt{\frac{m+9}{m+6}}, \quad \text { if } n>5 \text {. }
\end{aligned}
$$

Proof. We partition the size of $G$ into edges of the type $\left(S_{u}, S_{\nu}\right)$, where $u \nu \in E(G)$ as shown in Tables 2, 3, 4, and 5 for the case $n=3, n=4, n=5$, and $n>5$, respectively.

We know that

$$
A B C_{4}(G)=\sum_{u \nu \in E(G)} \sqrt{\frac{S_{u}+S_{\nu}-2}{S_{u} S_{\nu}}} .
$$

Hence we get the required results by using Tables $2-5$. 
Table 2. The size partition of $G$ for $n=3$

\begin{tabular}{|c|c|c|c|c|}
\hline $\begin{array}{c}\left(S_{u}, S_{\nu}\right), \text { where } \\
u \nu \in E(G)\end{array}$ & $(m+6, m+6)$ & $\left(m^{2}+5, m^{2}+5\right)$ & $\left(m^{2}+5, m+6\right)$ & $(m+6,6)$ \\
\hline \begin{tabular}{c} 
Number of edges \\
\hline
\end{tabular} & $m$ & $\frac{m(m-1)}{2}$ & $2 m$ & $2 m$ \\
\hline
\end{tabular}

Table 3. The size partition of $G$ for $n=4$

\begin{tabular}{|c|c|c|c|c|}
\hline $\begin{array}{c}\left(S_{u}, S_{\nu}\right), \text { where } \\
u \nu \in E(G)\end{array}$ & $(m+6, m+6)$ & $\left(m^{2}+5, m^{2}+5\right)$ & $\left(m^{2}+5, m+6\right)$ & $(m+6,5)$ \\
\hline Number of edges & $m$ & $\frac{m(m-1)}{2}$ & $2 m$ & $2 m$ \\
\hline
\end{tabular}

Table 4. The size partition of $G$ for $n=5$

\begin{tabular}{|c|c|c|c|c|c|}
\hline $\begin{array}{c}\left(S_{u}, S_{\nu}\right), \text { where } \\
u \nu \in E(G)\end{array}$ & $(4,5)$ & $(m+6, m+6)$ & $\left(m^{2}+5, m^{2}+5\right)$ & $\left(m^{2}+5, m+6\right)$ & $(m+6,5)$ \\
\hline $\begin{array}{c}\text { Number of } \\
\text { edges }\end{array}$ & $2 m$ & $m$ & $\frac{m(m-1)}{2}$ & $2 m$ & $2 m$ \\
\hline
\end{tabular}


Theorem 2.3. Let $G$ be the line graph of the Jahangir graph $J_{n ; m}$. Then

$$
G A_{5}(G)=\left\{\begin{array}{cc}
m+\frac{m(m-1)}{2}+\frac{4 m \sqrt{\left(m^{2}+5\right)(m+6)}}{m^{2}+m+11} & \text { if } n=3 ; \\
& +\frac{4 m \sqrt{6 m+36}}{m+12},
\end{array}\right.
$$

Table 5. The size partition of $G$ for $n>5$

\begin{tabular}{|c|c|c|c|c|c|c|}
\hline $\begin{array}{c}\left(S_{u}, S_{\nu}\right), \\
\text { where } \\
u \nu \in E(G)\end{array}$ & $(4,4)$ & $(4,5)$ & $(m+6, m+6)$ & $\left(m^{2}+5, m^{2}+5\right)$ & $\left(m^{2}+5, m+6\right)$ & $(m+6,5)$ \\
\hline $\begin{array}{c}\text { Number of edges } \\
\text { m(n-5) }\end{array}$ & $2 m$ & $m$ & $\frac{m(m-1)}{2}$ & $2 m$ & $2 m$ \\
\hline
\end{tabular}

Proof. We know that

$$
G A_{5}(G)=\sum_{u \nu \in E(G)} \frac{2 \sqrt{S_{u} S_{\nu}}}{S_{u}+S_{\nu}}
$$

Hence we get the required results by using Tables 2-5. 
Theorem 2.4. Let $G$ be the line graph of the Jahangir graph $J_{n ; m}$. Then

$$
S(G)=\left\{\begin{array}{c}
\frac{m(m+6)^{6}}{(2 m+10)^{3}}+\frac{1}{2} \frac{m(m-1)\left(m^{2}+5\right)^{6}}{\left(2 m^{2}+8\right)^{3}} \\
+\frac{2 m\left(m^{2}+5\right)^{3}(m+6)^{3}}{\left(m^{2}+m+9\right)^{3}}+\frac{432 m(m+6)^{3}}{(m+10)^{3}}, \\
+\frac{2 m\left(m^{2}+5\right)^{3}(m+6)^{3}}{\left(m^{2}+m+9\right)^{3}}+\frac{250 m(m+6)^{3}}{(m+9)^{3}}, \\
\frac{m(m+6)^{6}}{(2 m+10)^{3}}+\frac{1}{2} \frac{m(m-1)\left(m^{2}+5\right)^{6}}{\left(2 m^{2}+8\right)^{3}}
\end{array} \quad \text { if } n=3 ;\right.
$$

Proof. We know that

$$
S(G)=\sum_{u \nu \in E(G)}\left(\frac{S_{u} S_{\nu}}{S_{u}+S_{\nu}-2}\right)^{3}
$$

Hence we get the required results by using Tables $2-5$.

\section{References}

[1] S. H. Bertz, The bond graph, Journal of the Chemical Society, Chemical Communications 16 (1981), 818-820.

DOI: http://dx.doi.org/10.1039/C39810000818

[2] I. Gutman, Edge versions of topological indices, I. Gutman and B. Furtula (Editors), Novel Molecular Structure Descriptors-Theory and Applications II, Univ. Kragujevac, Kragujevac, 3 (2010). 
[3] I. Gutman and E. Estrada, Topological indices based on the line graph of the molecular graph, J. Chem. Inf. Comput. Sci. 36(3) (1996), 541-543.

DOI: http://dx.doi.org/10.1021/ci950143i

[4] I. Gutman, L. Popovic, B. K. Mishira, M. Kuanar, E. Estrada and N. Guevara, Application of line graphs in physical chemistry: Predicting the surface tension of alkenes, J. Serb. Chem. Soc. 62(11) (1997), 1025-1029.

[5] I. Gutman and Z. Tomovic, On the application of line graphs in quantitative structure-property studies, J. Serb. Chem. Soc. 65(8) (2000), 577-580.

[6] I. Gutman and Z. Tomovic, Modeling boiling points of cycloalkanes by means of iterated line graph sequences, J. Chem. Inf. Comput. Sci. 41(4) (2001), 1041-1045.

DOI: http://dx.doi.org/10.1021/ci010006n

[7] A. Iranmanesh, I. Gutman, O. Khormali and A. Mahmiani, The edge versions of the Wiener index, MATCH Commun. Math. Comput. Chem. 61(3) (2009), 663-672.

[8] H. Wiener, Structural determination of paraffin boiling points, J. Am. Chem. Soc. 69(1) (1947), 17-20.

DOI: http://dx.doi.org/10.1021/ja01193a005

[9] I. Gutman and N. Trinajstic, Graph theory and molecular orbitals: Total $\varphi$-electron energy of alternant hydrocarbons, Chem. Phys. Lett. 17(4) (1972), 535-538.

DOI: https://doi.org/10.1016/0009-2614(72)85099-1

[10] R. Todeschini and V. Consonni, Handbook of Molecular Descriptors, Wiley-VCH, Weinheim, 2000.

$$
\text { DOI: http://dx.doi.org/10.1002/9783527613106 }
$$

[11] G. H. Fath-Tabar, Old and new Zagreb indices of graphs, MATCH Commun. Math. Comput. Chem. 65(1) (2011), 79-84.

[12] G. H. Shirdel, H. Rezapour and A. M. Sayadi, The hyper-Zagreb index of graph operations, Iran. J. Math. Chem. 4(2) (2013), 213-220.

DOI: http://dx.doi.org/10.22052/IJMC.2013.5294

[13] A. A. Dobrynin and A. A. Kochetova, Degree distance of a graph: A degree analogue of the Wiener index, J. Chem. Inf. Comput. Sci. 34(5) (1994), 1082-1086.

[14] I. Gutman, Selected properties of the Schultz molecular topological index, J. Chem. Inf. Comput. Sci. 34(5) (1994), 1087-1089.

[15] M. Randic, On characterization of molecular branching, Journal of the American Chemical Society 97(23) (1975), 6609-6615.

DOI: http://dx.doi.org/10.1021/ja00856a001 
[16] B. Bollobas and P. Erdos, Graphs of extremal weights, Ars Combin. 50 (1998), 225-233.

[17] E. Estrada, L. Torres, L. Rodriguez and I. Gutman, An atom-bond connectivity index: Modelling the enthalpy of formation of alkanes, Indian J. Chem. 37(10) (1998), 849-855.

[18] B. Furtula, A. Graovac and D. Vukicevic, Atom-bond connectivity index of trees, Disc. Appl. Math. 157(13) (2009), 2828-2835.

DOI: https://doi.org/10.1016/j.dam.2009.03.004

[19] B. Zhou and N. Trinajstic, On general sum-connectivity index, J. Math. Chem. 47(1) (2010), 210-218.

DOI: https://doi.org/10.1007/s10910-009-9542-4

[20] B. Zhou and N. Trinajstic, On a novel connectivity index, J. Math. Chem. 46(4) (2009), 1252-1270.

DOI: https://doi.org/10.1007/s10910-008-9515-z

[21] B. Zhou and N. Trinajstic, On general sum-connectivity index, Journal of Mathematical Chemistry 47(1) (2010), 210-218.

DOI: https://doi.org/10.1007/s10910-009-9542-4

[22] D. Vukicevic and B. Furtula, Topological index based on the ratios of geometrical and arithmetical means of end-vertex degrees of edges, J. Math. Chem. 46(4) (2009), 1369-1376.

DOI: https://doi.org/10.1007/s10910-009-9520-x

[23] H. Hua and S. Zhang, Relations between Zagreb coindices and some distance-based topological indices, MATCH Commun. Math. Comput. Chem. 68(1) (2012), 199-208.

[24] M. Ghorbani and M. Ghazi, Computing some topological indices of triangular benzenoid, Digest Journal of Nanomaterials and Biostructures 5(4) (2010), 1107-1111.

[25] M. Ghorbani and M. A. Hosseinzadeh, Computing $\mathrm{ABC}_{4}$ index of nanostar dendrimers, Optoelectronics and Advanced Materials: Rapid Communications 4(9) (2010), 1419-1422.

[26] M. Ghorbani and M. Jalali, Computing a new topological index of nano structures, Digest Journal of Nanomaterials and Biostructures 4(4) (2009), 681-685.

[27] A. Graovac, M. Ghorbani and M. A. Hosseinzadeh, Computing fifth geometricarithmetic index for nanostar dendrimers, J. Math. Nanosci. 1(1-2) (2011), 33-42.

DOI: http://dx.doi.org/10.22061/JMNS.2011.461

[28] B. Furtula, A. Graovac and D. Vukicevic, Augmented Zagreb index, J. Math. Chem. 48(2) (2010), 370-380.

DOI: https://doi.org/10.1007/s10910-010-9677-3 
[29] S. M. Hosamani, Computing Sanskruti index of certain nanostructures, Journal of Applied Mathematics and Computing 54(1-2) (2017), 425-433.

DOI http://dx.doi.org/10.1007/s12190-016-1016-9

[30] I. Gutman, B. Furtula, Z. K. Vukicevic and G. Popivoda, On Zagreb indices and coindices, MATCH Commun. Math. Comput. Chem. 74(1) (2015), 5-16.

[31] I. Gutman and K. Ch. Das, The first Zagreb index 30 years after, MATCH Commun. Math. Comput. Chem. 50(1) (2004), 83-92.

[32] K. Ali, E. T. Baskoro and I. Tomescu, On the Ramsey Number of Paths and Jahangir Graph $J_{3, m}$, The 3rd International Conference on 21st Century Mathematics, GC University Lahoor, Pakistan, 2007. 